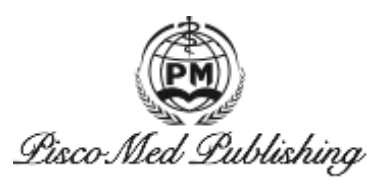

Review Article

\title{
Research and development of civil engineering intelligent structure system
}

\author{
Hongying Xu \\ Yuanxingming Construction Engineering Co., Ltd. Anhui Province, China; hongying@163.com
}

Abstract: With the fast-paced development of China's economy, science and technology have made rapid progress, as well as the research on civil engineering construction. In particular, civil engineering intelligent structure system has evolved into one of the world's leading disciplines and is also the future development trend, attracting extensive attention and research from professionals in this field. This paper mainly analyzes the research and current development of civil engineering intelligent structure system, and gives reasonable suggestions based on the main problems, so as to provide some references for better development and progress of this technology.

Keywords: Civil engineering; intelligent structure; development; research

Received: December 3, 2019 Accepted: December 22, 2019 Published: December 27, 2019

\section{Introduction}

China is with a vast territory, complicated geological conditions and many different tectonic plates, which makes it one of the countries with frequent natural disasters like earthquakes. However, earthquake harms civil engineering structure system most seriously, and also endanger people's life and property safety. Civil engineering comprises many basic constructions, which play an important role in people's production and life. Therefore, it is an important issue to be paid attention to that how we can improve the ability of civil engineering structure to resist natural disasters such as earthquakes and improve its safety and stability. Based on this consideration, it is more important for us to study and develop intelligent structure system. Starting with the components and related materials of civil engineering intelligent structure system, this paper discusses and studies it, and then gives corresponding solutions to the existing problems.

\section{Application status of civil engineering intelligent structure system}

Generally, civil engineering intelligent structure system looks like a bionic structure system, which aims to make civil structures more intelligent. Because of the progress and development of society, people's requirements for building structures are also constantly improving and the development of civil engineering intelligent structure system is the trend of the times. Usually in current engineering applications, intelligent structure system is mainly concentrated in the following aspects: structural damage detection and structural health monitoring. The technologies commonly used in modern building detection include vision and acoustic emission technology, ultrasonic technology and nondestructive testing technology. However, these can only be limited to buildings. When buildings are put into use, the detection in these aspects is often neglected,

\footnotetext{
Copyright (C) 2019 Hongying Xu

doi: $10.18282 /$ ice.v2i1.305

This is an open-access article distributed under the terms of the Creative Commons Attribution Non-Commercial License

(http://creativecommons.org/licenses/by-nc/4.0/), which permits non-commercial use, distribution, and reproduction in any medium, provided the original work is properly cited.
} 
resulting in overall degradation and untimely construction. However, a series of self-diagnoses, like the self-recognition of intelligent structure and detection of new sensing elements, not only are sensitive to injury occurrence, but also can effectively monitor the degree of injury, such as the use of real-time detection in the construction of optical fiber technology and semiconductor equipment, which is .

\section{Components and materials of intelligent structure system}

Generally speaking, the intelligent structure system consists of three parts: sensor, signal processor and controller. The design principle is based on bionics and realizes the collection, transmission and processing of relevant internal information of building structure according to the perception mode and recognition mode of organisms to the external environment. When the system collects dangerous signals from the outside, it will transmit them to the signal processing module for analysis and processing, and then the processing results will be feed back to the controller module, which can adjust the civil engineering structure, thus resisting external earthquake risks and eliminating hidden dangers. Materials that make up an intelligent system can generally be divided into two categories. First, materials used to realize control functions are shape memory materials, piezoelectric materials and various functional gels. The series of materials are mainly used to realize the relevant functions of the controller in the system, whose own characteristics can change along with the changes of various external environmental indicators. For example, they can change their characteristics of shape, structure and frequency with the changes of external temperature and magnetic field strength, so as to adapt to the outside world and reduce the damage to themselves. Secondly, sensing materials are mainly used to realize sensor-related functions in the system, such as strain synthetic metals, materials with automatic healing characteristics and optical fibers.

\section{Specific application of intelligent structure system in civil engineering construction}

\subsection{Specific application of shape memory synthetic metals}

The intelligent control system of civil engineering structure system is mainly composed of signal processor, sensor and controller, which is consistent with the principle of bionics. It can simulate the potential danger that will happen soon, and affect positively on the safety of building structure to make corresponding adjustment. For example, when the structure of the sensor is found to have potential safety hazards, the controller will receive the information related to the safety risks and then adjust the civil engineering structure, thus achieving the purpose of intelligently processing the safety risks. The application of intelligent structure system greatly affects the maintenance and design of civil engineering. When an earthquake occurs, a large amount of energy will be generated in a short period of time, which will cause deformation of buildings. The application of shape memory synthetic metals, based on the adoption of its particularly strong characteristics of phase change recovery, can offset the deformation energy to a large extent, thus improving the building's ability to resist earthquake disasters. Its mechanism is to receive the structural deformation of buildings by use of energy dissipators, and then offset the energy generated by the earthquake to achieve the seismic effect.

\subsection{Specific application of piezoelectric materials}

Currently, application of piezoelectric materials in civil engineering can not only control static deformation and noise, but also realize functions like adaptive recovery, safety evaluation and health monitoring, which is helpful to improve risk resistance and seismic resistance. Intelligent materials are mainly divided into two categories, one is driving material, such as shape memory materials and magnetorheological materials. The main characteristic of these materials is to adjust by use of temperature, electric field, and magnetic field's shape, location and frequency, and form a certain adaptive function. The other is sensor element, whose characteristic is the physical function that motivates the perception of strength and strain capacity of the material through the internal and external structures, so it is also 
called sensitive material. These materials include optical fibers, sub-polymer alloys and piezoelectric ceramics, among which optical fibers is the most important in current applications. The material can change its shape, position and frequency according to the changes of temperature, electric field and magnetic field, and form certain mechanical properties, which enables the preparation of special materials and the synthesis of driving elements. Piezoelectric cone technology is generally adopted in buildings to improve the initiative of anti-seismic measures, and good results have been achieved. In the construction process, the concrete structure is mixed with healing materials (such as polypropylene fiber) in a certain proportion, so that the building structure can be quickly recovered when damaged by earthquakes. This is the best way to overcome the damage of concrete structure at present. However, this technology is still under improvement and will definitely have a lot of application space when being mature.

\subsection{Specific application of optical fibers}

In the system, sensors that actively control seismic response are generally made of optical fibers, which can diagnose structural health of buildings. Its mechanism is adding a certain optical fiber into the normal concrete structure in the process of construction to form conductive components, thus realizing related functions such as self-inspection, observation and control. Indicators of the building structure include the deformation, damage, strength, vibration are monitored in real time, thereby forming an intelligent structure system to enable civil engineering buildings to self detect and automatically recover. With the rapid development of intelligent materials technology and the changeable development direction of civil engineering intelligent structure, it is necessary for us to combine the material advantages of intelligent buildings and carry out corresponding civil engineering intelligent structure design so as to make it become intelligent and structured intelligent material of system engineering. Development of intelligent system lays a solid foundation for the civil engineering intelligent structure system and provides a good developing opportunity for civil engineering intelligent structure system. At present, as China's intelligent structure system has made great progress, it is necessary to actively promote the application of civil engineering intelligent structure system and the development of intelligent civil engineering.

\section{Research and future development direction of intelligent structure system}

\subsection{Intelligent driving technology}

The mechanism of this technology is that when the external environment of the building changes, the intelligent driving components can change their own characteristics accordingly to adapt to the new external environment, thus ensuring the safety and stability of the building structure. Therefore, the research and application of intelligent driving technology need to be increased, which can be started from three aspects. First, improve the integration of driving and main materials, and then enhance their strength and effectiveness. Second, try to adopt materials with large elasticity and strong impact resistance as driving materials. Third, carry out relevant research continuously to improve its response frequency and speed.

\subsection{Enhance the research on intelligent integration process}

The control system of structure is like animals' brain, which coordinates and controls various actions and functions of the organisms. Therefore, in order to improve the control performance of civil engineering intelligent structure system, it is necessary to intensify the research and development of its intelligent integration in the future, and strive to realize the cooperative control of building structure and deformation. Civil engineering intelligent structure system can promote the development of integrated intelligent control technology and intelligent integrated processing, and realize optimal control of all components of intelligent structure. All elements in the intelligent structure are in a semi-closed state. By analyzing various risk factors, the application of intelligent integrated control technology enables the system to enhance variability, and solves the problem of stability. The system can be controlled to avoid various intelligent components caused by sudden start-up of unstable structure, thus making the system run more smoothly. 


\subsection{Enhance the research on information transmission and processing}

The main auxiliary system of civil engineering intelligent structure system is information processing and transmission, among which information processor and transmitter occupy most of the functions. It can transmit information of civil structure damage timely and accurately to the control system for intelligent recovery. Due to the limitation of calculation process and data lines, the processing and transmission of data is delayed. To speed up the transmission speed of information processing functions, it is necessary to combine the processor with transmitter. In the whole system, the transmission and processing of information are mainly completed through signal transmitters and controllers, which play the functions of processing, uploading and releasing information, and connecting various devices to form an organic whole. Therefore, in order to improve the overall performance of the system, it is necessary to intensify research and development in this area.

\section{Conclusion}

To sum up, civil engineering intelligent structure system is the main technology currently being used in the construction industry, and is also an important research direction in the future, which can improve the safety of buildings to some extent. In the future, human science and technology will continue to be improved along with many new materials being discovered. Therefore, we need to actively increase research efforts, and continuously optimize the intelligent structure system in order to improve the safety of buildings, enhance the earthquake resistance of buildings, and ensure the safety of people's lives and property.

\section{References}

1. Wang ZL. Application of slope support technology in civil engineering construction [J]. Henan Building Materials 2020; (01): 20.

2. Cao HS. Study on effective control of civil engineering management and engineering cost $[\mathrm{J}]$. Henan Building Materials 2020; (01): 68-69.

3. Chen X. Discussion on key issues of construction management in civil engineering [J]. Henan Building Materials 2020; (01): 72-73. 